\title{
Weinberg power counting and the quark determinant at small chemical potential
}

\author{
E. S. Fraga ${ }^{1}$ and C. Villavicencio ${ }^{2}$ \\ ${ }^{1}$ Instituto de Física, Universidade Federal do Rio de Janeiro, \\ C.P. 68528, Rio de Janeiro, RJ 21945-972, Brasil \\ ${ }^{2}$ Facultad de Física, Pontifcia Universidad Católica de Chile, Casilla 306, Santiago 22, Chile
}

\begin{abstract}
We construct an effective action for QCD by expanding the quark determinant in powers of the chemical potential at finite temperature in the case of massless quarks. To cut the infinite series we adopt the Weinberg power counting criteria. We compute the minimal effective action $\left(\sim p^{4}\right)$, expanding in the external momentum, which implies the use of the hard thermal loop approximation. Our main result is a gauge invariant expression for the phase $\theta$ of the functional determinant in QCD, and recovers dimensional reduction in the high-temperature limit. We compute, analytically, $\left\langle\theta^{2}\right\rangle$ in the range of $p \ll 2 \pi T$, including perturbative and nonperturbative contributions, the latter treated within the mean field approximation. Implications for lattice simulations are briefly discussed.
\end{abstract}

PACS numbers: 11.10.Wx, 11.15.-q, 12.39.-x, 25.75.Nq

\section{INTRODUCTION}

There has been an increasing interest in the last few years in the sign problem or phase problem in QCD [1]. For a finite chemical potential, $\mu$, the fermion determinant matrix is non-positive definite, so it is not possible to perform Monte Carlo simulations in the usual fashion [2, 3]. Nevertheless, the Glasgow method [4] and rewheighting techniques [5] have provided great advances in the description of phase transitions on the lattice, considering a set of parameters near the transition line.

There is special interest in the region of high temperature and low chemical potential, since it corresponds to the sector of the phase diagram of strong interactions probed by high-energy heavy-ion collision experiments [6]. In this regime it is possible to expand the fermion determinant in powers of $\mu / \Lambda$, where $\Lambda$ is some mass scale related to the temperature, $\Lambda \sim T[7,11]$. In association with the ones mentioned previously, this technique is very convenient and successful to describe this region. Besides, several other complementary approaches were proposed with the intent to shed some light on the sign problem [12 17].

In this paper we investigate the small chemical potential sector of the QCD phase diagram. In particular, we present a scheme that is valid for a wide range of temperatures in the soft region. Methods that are based on an expansion in $\mu$ always have to resort to approximations to compute the coefficients of the series. For this purpose, there are numerical approximation techniques that yield good results but do not allow for a deeper analytic study, the most frequently used being the random noise method (see, e.g., Ref. [18]). Analytic treatments are usually restricted to very high temperatures. For instance, the dimensional reduction effective action [19 24] is valid for $T \gtrsim 2 T_{c}$, where $T_{c}$ is the critical temperature. A complete study of the pressure within perturbative QCD for all values of the temperature and chemical potential can be found in Ref. [25] where the authors make use of the hard thermal loop, hard dense loop and dimensional reduction approximations.
Here we start from a different perspective: instead of expanding all quantities and desired observables in powers of $\mu / \Lambda$, the idea is to keep the relevant terms in the effective action according to the Weinberg power counting criteria [26]. In what follows we construct an effective action for QCD by expanding the quark determinant in powers of the chemical potential at finite temperature in the case of massless quarks. We compute the minimal effective action expanding in the external momentum up to order $\sim p^{4}$ in power counting. In practice, the momentum expansion performed here is equivalent to the hard thermal loop (HTL) approximation [27-29]. Our main result is a gauge invariant expression for the phase angle $\theta(\mu)$ of the functional determinant in QCD, which can be written as $\operatorname{det} M(\mu)=|\operatorname{det} M(\mu)| e^{i \theta(\mu)}$. An interesting analysis of the angle $\theta$ has been recently performed using the random matrix framework [16, 17].

As a first test of our method, we recover the dimensional reduction approximation in the limit of high temperature. As a second step, we compute analytically $\left\langle\theta^{2}\right\rangle$ in the range of $p \ll 2 \pi T$, keeping nonzero mode contributions in the effective action. In this calculation, we include perturbative and nonperturbative contributions, treating the latter in the mean field approximation. Implications for lattice simulations are also discussed.

This paper is organized as follows. In Sec. II. we develop the general expansion for the fermionic determinant, present the Feynman rules and the relevant diagrams, settling the framework. In Sec. III. we discuss the power counting hierarchy in order to be able to cut the series and compute the minimal effective action. As a first test of our framework, we also reobtain the result from dimensional reduction in the limit of high temperature, including the non-zero mode terms. In Section IV we present the calculation of $\left\langle\theta^{2}\right\rangle$ in the range of $p \ll 2 \pi T$. Section V contains our conclusions. 


\section{THE EXPANSION}

The generating functional for QCD with massless quarks at finite chemical potential is defined, in euclidean space, as

$$
\mathcal{Z}=\int \mathcal{D} G \operatorname{det}\left(-i \not D+i \mu \gamma_{4}\right) e^{-S_{\mathrm{YM}}[G]}
$$

where $G$ are the gluon fields, also present in the covariant derivative $D_{\mu}=\partial_{\mu}-i G_{\mu}$, and $S_{\mathrm{YM}}$ is the Yang-Mills (YM) action. We can expand the fermion determinant in powers of the chemical potential assuming that $\mu<$ $\Lambda \sim T$ :

$$
\begin{aligned}
& \operatorname{det}\left(-i \not D+i \mu \gamma_{4}\right)= \\
& \operatorname{det}(-i \not D) \exp \left\{-N_{f} \sum_{s=1}^{\infty} \frac{(-i \mu)^{s}}{s} \int_{\beta} d y_{1} \cdots d y_{s}\right. \\
& \left.\quad \operatorname{Tr} \gamma_{4} S\left(y_{2}, y_{1}\right) \gamma_{4} S\left(y_{3}, y_{2}\right) \cdots \gamma_{4} S\left(y_{1}, y_{n}\right)\right\}
\end{aligned}
$$

where $\int_{\beta} d y \equiv \int_{0}^{\beta} d y_{4} \int d^{3} y, S\left(y_{b}, y_{a}\right)$ is the dressed fermion propagator, which can be expressed as a series in powers of the gauge field and the free fermion propagator using the self-consistent relation

$$
S(x, y)=S_{F}(x-y)-\int_{\beta} d z S(x, z) \not(z) S_{F}(z-y) .
$$

The expansion, then, will contribute to additional terms in the effective action $S_{\text {eff }}=S_{\mathrm{YM}}+\sum_{n, s} S^{(n, s)}$. The new terms, expressed in momentum space, are of the form

$$
\begin{aligned}
S^{(n, s)}= & \mu^{s} \mathcal{f} d p_{1} \cdots d p_{n}(2 \pi)^{4} \delta\left(p_{1}+\cdots+p_{n}\right) \\
& \Gamma_{\mu_{1} \cdots \mu_{n}}^{(s, n)}\left(\left\{p_{i}\right\}\right) \operatorname{tr} \tilde{G}_{\mu_{1}}\left(p_{1}\right) \cdots \tilde{G}_{\mu_{n}}\left(p_{n}\right),
\end{aligned}
$$

where the last integral denotes also the sum over bosonic Matsubara frequencies $£ d p \equiv T \int \frac{d^{3} p}{(2 \pi)^{3}} \sum_{p_{4}=2 n_{p} \pi T}$, and $2 \pi T \delta\left(p_{4}\right)=\delta_{n_{p}, 0}$. In this way, one obtains a positivedefinite fermion determinant, and the contribution from the chemical potential will be part of an effective gluon action.

The Feynman rules in momentum space for calculating the different effective vertices are almost the same as the usual ones. The difference is that all of the operators between chemical potential insertions must be transposed in order. Figure 1 shows a general diagram with chemical potential and gluon insertions. To construct a diagram for a vertex with $n$ gluons and $s$ chemical potential insertions, one puts $-t_{a_{i}} \gamma_{\mu_{i}}$ for any gluon insertion, $-i \gamma_{4}$ for any chemical potential insertion, and divides by the symmetry factor $s$.

Between chemical potential insertions, the order of the operator must be transposed considering momentum conservation. In a piece of the effective vertex shown in Fig.

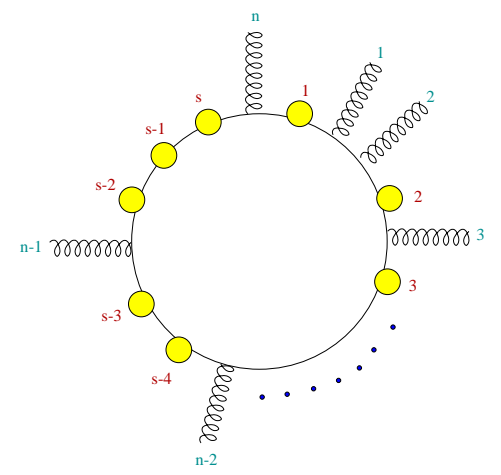

FIG. 1: General diagram for the construction of the effective vertices. The small circles correspond to chemical potential insertions.

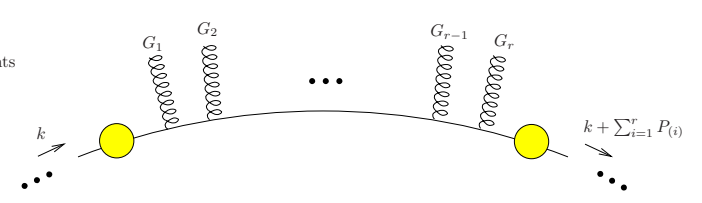

FIG. 2: A set of operators between two chemical potential insertions.

2, the integrand must be written as

$$
\begin{aligned}
& \left(-i \gamma_{4}\right)\left[\tilde{S}_{F}(k)\left(-t_{a_{1}} \gamma_{\mu_{1}}\right) \tilde{S}_{F}\left(k+p_{1}\right) \cdots\right. \\
& \left.\cdots\left(-t_{a_{r}} \gamma_{\mu_{r}}\right) \tilde{S}_{F}\left(k+p_{1}+\cdots+p_{r}\right)\right]^{(t)}\left(-i \gamma_{4}\right)
\end{aligned}
$$

where the exponent $(t)$ in the brackets is a reminder to transpose the order of the operators: $\left[\mathcal{O}_{1} \mathcal{O}_{2} \cdots \mathcal{O}_{r-1} \mathcal{O}_{r}\right]^{(t)}=\mathcal{O}_{r} \mathcal{O}_{r-1} \cdots \mathcal{O}_{2} \mathcal{O}_{1}$

Finally, one takes the trace over gamma matrices and color group representation, integrating over internal fermionic momentum (odd Matsubara frequencies). The sum of all diagrams will produce the effective vertices $\Gamma^{(n, s)}$, which will be invariant under any cyclic change in the set of indices $\mu_{i}, a_{i}, p_{i}$ (or $x_{i}$ in the case of configuration space).

\section{THE MINIMAL EFFECTIVE ACTION}

Now, we need a criteria to cut the series. For lowenergy effective theories, one can consider the Weinberg power counting [26], which uses the argument that all mass parameters (external momentum, chemical potential and gluon fields) must be less than a certain scale that is proportional to the temperature. This approach has provided a very successful description within chiral effective models of QCD. Although this is not exactly the case here, it is reasonable that for low-energy processes at high temperature typical values of the operators mentioned above, as well as the chemical potential, can be considered to be in a region of the same order or smaller 
than the scale. Then, assuming $\mu \sim G \sim p$, and expanding the effective Lagrangean in soft modes, we can cut it at a given order in powers of the momentum scale.

In the case of Yang-Mills theories, this soft-mode expansion for high temperatures corresponds to the HTL approximation. The minimal action must be of order $(p)^{4}$. So, applying the power counting criteria, the minimal effective action is given by $S_{\text {eff }}^{\min }=S_{\mathrm{YM}}+S^{(0,2)}+$ $S^{(0,4)}+\left[S^{(2,2)}+S^{(3,1)}\right]_{\mathcal{O}(p)^{0}}$, where the indices $(n, s)$ are defined in Eq. (4), and the last two terms are expanded in momentum up to zeroth order which leads to the appearance of functions of $|\boldsymbol{p}| / p_{4}$.

The whole series of gauge fields is gauge invariant at each order in the expansion in $\mu$, i.e. $\sum_{n} S^{(n, s)}$ is gauge invariant for all values of $s$ as can be seen directly from Eq. (2). Since the terms in the sum are traces containing dressed propagators, which are gauge invariant, every term in the sum is gauge invariant. Moreover, the minimal effective vertices that we need satisfy Ward identities of the form

$$
p_{\mu} \Gamma_{\mu \nu}^{(2,2)}(p)=0,
$$

$$
p_{\mu} \Gamma_{\mu \nu \sigma}^{(3,1)}(p, q, r)=\Gamma_{\nu \sigma}^{(2,1)}(r)-\Gamma_{\nu \sigma}^{(2,1)}(q),
$$

and analogous relations obtained by changing cyclical indices and arguments. Equation (7) vanishes, since $\Gamma^{(2,1)}=0$. As is well known, HTL preserves the Ward identities.

The nonvanishing diagrams for the vacuum contributions $\Gamma^{(s, 0)}$ are the known vacuum corrections to the thermodynamic potential

$$
\Gamma^{(0,2)}=-N_{c} N_{f} \frac{T^{2}}{6}, \quad \Gamma^{(0,4)}=-N_{c} N_{f} \frac{1}{12 \pi^{2}} .
$$

For $s>4$ all contributions vanish, as was demonstrated in Ref. [30]. The next nonvanishing term has the form of the polarization tensor in the HTL approximation

$$
\Gamma_{\mu \nu}^{(2,2)}(p)=\frac{N_{f}}{2 \pi^{2}} \int \frac{d \Omega}{4 \pi}\left[\frac{i p_{4}}{\hat{k} \cdot p} \hat{k}_{\mu} \hat{k}_{\nu}+\delta_{\mu 4} \delta_{\nu 4}\right],
$$

with the lightlike four-vector $\hat{k}=(\hat{\boldsymbol{k}}, i)$. Finally, the vertex components which correspond to $i \theta$ are

$$
\begin{array}{r}
\Gamma_{\mu \nu \rho}^{(3,1)}(p, q, r)=\frac{i N_{f}}{6 \pi^{2}} \int \frac{d \Omega}{4 \pi}\left\{2 \delta_{\mu 4} \delta_{\nu 4} \delta_{\rho 4}-\right. \\
\frac{p_{4}}{\hat{k} \cdot p}\left[\hat{k}_{\mu} \delta_{\nu \rho}-6 \hat{k}_{\mu} \hat{k}_{\nu} \hat{k}_{\rho}+2 i\left(\hat{k}_{\mu} \hat{k}_{\nu} \delta_{\rho 4}+\hat{k}_{\nu} \hat{k}_{\rho} \delta_{\mu 4}+\hat{k}_{\rho} \hat{k}_{\mu} \delta_{\nu 4}\right)\right] \\
-\frac{q_{4}}{\hat{k} \cdot q} \hat{k}_{\nu} \delta_{\rho \mu}-\frac{r_{4}}{\hat{k} \cdot r} \hat{k}_{\rho} \delta_{\mu \nu}+2 i\left(\frac{p_{4}}{\hat{k} \cdot p}\right)^{2} \hat{k}_{\mu} \hat{k}_{\nu} \hat{k}_{\sigma} \\
+\frac{q_{4}}{\hat{k} \cdot q \hat{k} \cdot p}\left[2 i q_{4} \hat{k}_{\mu} \hat{k}_{\nu} \hat{k}_{\rho}+\left(q_{\nu} \hat{k}_{\rho}-r_{\rho} \hat{k}_{\nu}\right) \hat{k}_{\mu}+\left(q_{\mu}-r_{\mu}\right) \hat{k}_{\nu} \hat{k}_{\rho}\right] \\
\quad+\frac{r_{4}}{\hat{k} \cdot r \hat{k} \cdot p}\left[2 i r_{4} \hat{k}_{\mu} \hat{k}_{\nu} \hat{k}_{\rho}+\left(r_{\rho} \hat{k}_{\nu}-q_{\nu} \hat{k}_{\rho}\right) \hat{k}_{\mu}+\left(r_{\mu}-q_{\mu}\right) \hat{k}_{\nu} \hat{k}_{\rho}\right] \\
\left.+\left[\frac{q_{4}\left(q^{2}-r^{2}\right)}{\hat{k} \cdot q(\hat{k} \cdot p)^{2}}-\frac{q_{4} q^{2}}{(\hat{k} \cdot q)^{2} \hat{k} \cdot p}+\frac{r_{4}\left(r^{2}-q^{2}\right)}{\hat{k} \cdot r(\hat{k} \cdot p)^{2}}-\frac{r_{4} r^{2}}{\left.(\hat{k} \cdot r)^{2} \hat{k} \cdot p\right]}\right] \hat{k}_{\mu} \hat{k}_{\nu} \hat{k}_{\rho}\right\}
\end{array}
$$

The dimensional reduction approximation can be obtained directly from the last expressions of the effective vertices by simply considering the case in which $|\boldsymbol{p}| \ll 2 \pi T$. In order to expand in powers of the external momentum we have to separate the zero mode from the other modes in the gluon fields $G_{\mu}=A_{\mu}+B_{\mu}$, with

$$
\begin{aligned}
& A_{\mu}(\boldsymbol{x})=T \int \frac{d^{3} p}{(2 \pi)^{3}} e^{i \boldsymbol{p} \cdot \boldsymbol{x}} \tilde{G}_{\mu}(\boldsymbol{p}, 0), \\
& B_{\mu}(x)=T \sum_{p_{4} \neq 0} \int \frac{d^{3} p}{(2 \pi)^{3}} e^{i p \cdot x} \tilde{G}_{\mu}(p),
\end{aligned}
$$

$A_{\mu}$ being the field corresponding to the zero mode. The lowest order contribution in the expansion $(p \rightarrow 0)$ is given by

$$
\begin{aligned}
& S^{(2,2)}=\frac{\mu^{2} N_{f}}{2 \pi^{2}} \int_{\beta} d x \operatorname{tr}\left[A_{4}^{2}-\frac{1}{3} \boldsymbol{B}^{2}\right], \\
& S^{(3,1)}=\frac{i \mu N_{f}}{3 \pi^{2}} \int_{\beta} d x \operatorname{tr}\left[A_{4}^{3}+A_{4} B_{4}^{2}\right]
\end{aligned}
$$

If we set $B=0$, we recover the dimensional reduction effective action at tree level [23, 24]. The usual effective action for the high-temperature regime is constructed through loops integrating the $B$ fields. However, this is done perturbatively, and this is not the regime in which we are interested. The fact of including $B$ as a nonperturbative field (except for the power counting) in principle will enhance the range of validity in temperature to values lower than in the case of just considering an infinite temperature expansion. In this sense, our frame- 
work goes beyond dimensional reduction, and can probe temperatures closer to $T_{c}$.

\section{IV. $\left\langle\theta^{2}\right\rangle$ AT HIGH TEMPERATURE}

The phase angle $\theta$ of the complex functional determinant is a crucial quantity for lattice simulations in QCD. The knowledge of $\theta$ allows for the separation of the functional integral into two different regions: $|\theta| \lessgtr \pi / 2$. Nevertheless this is not a simple task, since it is not restricted to values of $-\pi<\theta<\pi$ but seems to increase with the volume [31]. The calculation of the average phase

$$
\left\langle e^{2 i \theta}\right\rangle=\left\langle\frac{\operatorname{det}\left(-i \not D+i \mu \gamma_{4}\right)}{\operatorname{det}\left(-i \not D+i \mu \gamma_{4}\right)^{\dagger}}\right\rangle
$$

gives a measure of how problematic could be the phase in lattice simulations. As this quantity must vanish in the thermodynamic limit, it should be evaluated in a finite volume (otherwise the average phase above vanishes as soon as $\mu$ is nonzero). The random matrix aproximation [16] yields a vanishing result for $\mu>m_{\pi} / 2$. One can use our effective expression for the phase to compute the average phase factor in Eq. (15). If one expands the exponential in powers of the angle, the relevant term will be $\sim\left\langle\theta^{2}\right\rangle$, since the average of the phase must be real. In particular, the determination of $\left\langle\theta^{2}\right\rangle_{P}$, the average of the angle as a function of the plaquette, is an important ingredient in order to localize the critical line in the temperature-number density phase diagram of QCD [31].

In our framework, we have $S^{(3,1)}=i \theta$. Writing the fields in the form

$$
\begin{aligned}
& A_{\mu}^{a}(\boldsymbol{x})=T \int_{0}^{\beta} d x_{4} G_{\mu}^{a}(x), \\
& B_{\mu}^{a}(x)=G_{\mu}^{a}(x)-T \int_{0}^{\beta} d x_{4} G_{\mu}^{a}(x),
\end{aligned}
$$

we can use Eq. (14) to express $\theta$ in the high-temperature limit as

$$
\theta=\frac{\mu N_{f}}{12 \pi^{2}} d^{a b c} T \int_{V} d^{3} x \int_{0}^{\beta} d x_{4} d y_{4} G_{\alpha}^{a}(y) G_{\beta}^{b}(x) G_{\gamma}^{c}(x),
$$

where $x=\left(\boldsymbol{x}, x_{4}\right), y=\left(\boldsymbol{x}, y_{4}\right)$ and $d^{a b c}$ are real and totally symmetric as usual. The expectation value of $\theta$ vanishes, so we compute the next power which can be expressed in terms of two-point correlation functions as

$$
\begin{aligned}
& \left\langle\theta^{2}\right\rangle \approx \frac{\mu^{2} T^{2} N_{f}^{2}\left(N_{c}^{2}-4\right)}{\left(12 \pi^{2}\right)^{2} N_{c}\left(N_{c}^{2}-1\right)^{2}} \int_{0}^{\beta} d x_{4} d x_{4}^{\prime} d y_{4} d y_{4}^{\prime} \\
& \times \int_{V} d^{3} x d^{3} x^{\prime}\left[2\left\langle G_{4}^{a}(y) G_{4}^{a}\left(y^{\prime}\right)\right\rangle\left\langle G_{4}^{a}(x) G_{4}^{a}\left(x^{\prime}\right)\right\rangle^{2}\right. \\
& \left.+4\left\langle G_{4}^{a}(y) G_{4}^{a}\left(x^{\prime}\right)\right\rangle\left\langle G_{4}^{a}(x) G_{4}^{a}\left(y^{\prime}\right)\right\rangle\left\langle G_{4}^{a}(x) G_{4}^{a}\left(x^{\prime}\right)\right\rangle\right]
\end{aligned}
$$

where the expression is not exact since we allow for nonperturbative contributions, and we used $\left\langle G^{a} G^{b}\right\rangle \sim \delta^{a b}$.
If we separate the gluon field in a perturbative and a nonperturbative contribution, $G=G_{p}+G_{n p}$, with $\left\langle G_{p} G_{n p}\right\rangle=0$, there will be contributions to $\left\langle\theta^{2}\right\rangle$ coming from a purely perturbative term, a purely nonperturbative term and the crossed terms. The basic building blocks are the two-point functions for the field strengths, which we compute in the sequel.

For the perturbative case, we start with the HTL effective Lagrangian

$$
\begin{aligned}
\mathcal{L}_{g}= & \frac{1}{4 g^{2}}\left(G_{\mu \nu}^{a}\right)^{2}+\frac{1}{4 g^{2}}\left(\partial_{\mu} G_{\mu}^{a}\right)^{2} \\
& +\frac{m^{2}}{4 g^{2}} G_{\mu \alpha}^{a} \int \frac{d \Omega}{4 \pi} \frac{\hat{k}_{\alpha} \hat{k}_{\beta}}{(i \hat{k} \cdot D)^{2}} G_{\beta \mu}^{a},
\end{aligned}
$$

where $m$ is the standard HTL effective mass [32]

$$
m^{2}=\frac{g^{2}}{6}\left[T^{2}\left(N_{f}+2 N_{c}\right)+\frac{3 N_{f} \mu^{2}}{\pi^{2}}\right] .
$$

Then, the fourth component of the gluon two-point function can be written, in the limit $p \ll 2 \pi T$, as

$$
\begin{array}{r}
\left\langle G_{4}^{a}(x)_{p} G_{4}^{b}\left(x^{\prime}\right)_{p}\right\rangle=\delta^{a b} g^{2} T \int \frac{d^{3} p}{(2 \pi)^{3}} e^{i \boldsymbol{p} \cdot\left(\boldsymbol{x}-\boldsymbol{x}^{\prime}\right)} \\
\times\left[\frac{1}{\boldsymbol{p}^{2}+m^{2}}+\sum_{n \neq 0} \frac{e^{i \omega_{n}\left(x_{4}-x_{4}^{\prime}\right)}}{\omega_{n}^{2}+\boldsymbol{p}^{2}}\right],
\end{array}
$$

where $\omega_{n}=2 n \pi T$ is the bosonic Matsubara frequency.

For the nonperturbative case it is convenient to use the Schwinger gauge, also known as fixed point or coordinate gauge [33], $x \cdot G=0$. Then, the gauge field can be expressed in terms of the field strength tensor:

$$
G_{\mu}^{a}(x)=\int_{0}^{1} d s s G_{\mu \alpha}^{a}(s x) x_{\alpha} .
$$

If we approximate the two-point function for the field strength by its mean field value, i.e.

$$
\left\langle G_{4 \alpha}^{a}(x) G_{4 \alpha}^{a}(y)\right\rangle \approx\left\langle G_{4 \alpha}^{a}(0) G_{4 \alpha}^{a}(0)\right\rangle \equiv-\left\langle\mathcal{E}^{2}\right\rangle,
$$

where $\mathcal{E}_{i}^{a}$ is the color electric field, we find

$$
\left\langle G_{4}^{a}(x)_{n p} G_{4}^{a}\left(x^{\prime}\right)_{n p}\right\rangle \approx-\frac{1}{12} \boldsymbol{x} \cdot \boldsymbol{x}^{\prime}\left\langle\mathcal{E}^{2}\right\rangle .
$$

Using the results above, the computation of $\left\langle\theta^{2}\right\rangle$ is long but straightforward. Collecting all terms, and assuming a large volume, we find

$$
\begin{aligned}
\left\langle\theta^{2}\right\rangle \approx & \frac{\mu^{2} N_{f}^{2}\left(N_{c}^{2}-4\right)}{\left(12 \pi^{2}\right)^{2} N_{c}}\left[\frac{\pi\left\langle\mathcal{E}^{2}\right\rangle^{2} g^{2}}{8\left(N_{c}^{2}-1\right) T}\left(\frac{R^{7}}{m^{2}}+\frac{R^{5}}{m^{4}}\right)\right. \\
& \left.-\frac{3\left\langle\mathcal{E}^{2}\right\rangle g^{4}}{32 \pi} \frac{R^{5}}{5 m}+F\left(\beta m, \beta \Lambda_{\overline{\mathrm{MS}}}\right) T g^{6} R^{3}\right],
\end{aligned}
$$

where $R$ is the radius of the system in spherical coordinates, $\Lambda_{\overline{\mathrm{MS}}}$ is the energy scale in the modified minimal 
subtraction (MS) scheme, and $F$ is an integral that can be computed numerically.

The last term is purely perturbative, whereas the other ones come from the mixed contribution. The purely nonperturbative contribution vanishes identically in the mean field approximation. Notice that, in the thermodynamic limit, the dominant contribution is the one proportional to $R^{7} \sim V^{7 / 3}$, so that $\left\langle\theta^{2}\right\rangle$ grows faster than quadratically with the volume, the other contributions being essentially irrelevant.

\section{CONCLUSIONS AND OUTLOOK}

We presented a well-defined and systematic procedure for computing the fermionic determinant in QCD at finite temperature and chemical potential. In the framework defined by the power counting method described above, we calculated exactly the minimal corrections to the effective action. This procedure can be systematically extended to higher orders.

We also computed explicitly $\left\langle\theta^{2}\right\rangle$ in the hightemperature limit and analyzed its volume dependence. In this limit, the dominant contribution comes naturally from the zero Matsubara mode, so that dimensional reduction is a good approximation. Although we do not present results beyond the mean field approximation for the gluon condensate, we expect that in that case, a new length scale associated with the nonuniformity of the condensate will compete with the scale $R$ but should not modify the picture appreciably in the limit considered in this work.

In this paper we proposed a new way of approaching the problem of determining the phase of the complex functional determinant in QCD at small chemical potential and relatively large temperatures, an important issue for lattice QCD thermodynamics. Our framework clearly contains, and goes beyond, the dimensional reduction approximation, being valid for temperatures closer to $T_{c}$. We believe that this analysis, complemented by previous existing results, might shed some light onto the phase transition in the region of high temperature and low density presumably probed by relativistic heavy-ion experiments, and perhaps indicate a simple way to handle the sign problem for temperature values closer to the critical temperature.

\section{Acknowledgment}

The authors acknowledge financial support from ANPCyT, CAPES, CLAF, CNPq, FAPERJ and FUJBUFRJ.
[1] Proceedings of Lattice 2009, PoS(LAT2009) (2009).

[2] E. Laermann and O. Philipsen, Ann. Rev. Nucl. Part. Sci. 53, 163 (2003).

[3] S. Hands, Prog. Theor. Phys. Suppl. 168, 253 (2007).

[4] I. M. Barbour and A. J. Bell, Nucl. Phys. B 372, 385 (1992).

[5] Z. Fodor and S. D. Katz, Phys. Lett. B 534, 87 (2002).

[6] Proceedings of Quark Matter 2009, Nucl. Phys. A 830, 1c-985c (2009).

[7] S. A. Gottlieb, W. Liu, D. Toussaint, R. L. Renken and R. L. Sugar, Phys. Rev. Lett. 59, 2247 (1987).

[8] S. Ejiri, C. R. Allton, S. J. Hands, O. Kaczmarek, F. Karsch, E. Laermann and C. Schmidt, Nucl. Phys. Proc. Suppl. 119, 538 (2003).

[9] C. R. Allton, S. Ejiri, S. J. Hands, O. Kaczmarek, F. Karsch, E. Laermann and C. Schmidt, Phys. Rev. D 68, 014507 (2003).

[10] R. V. Gavai and S. Gupta, Phys. Rev. D 71, 114014 (2005).

[11] C. R. Allton et al., Phys. Rev. D 71, 054508 (2005).

[12] P. de Forcrand and O. Philipsen, Nucl. Phys. B 642, 290 (2002).

[13] D. K. Hong and S. D. H. Hsu, Phys. Rev. D 68, 034011 (2003).

[14] M. Imachi, Y. Shinno and H. Yoneyama, Prog. Theor. Phys. 115, 931 (2006).

[15] K. Fukushima and Y. Hidaka, Phys. Rev. D 75, 036002 (2007).

[16] K. Splittorff and J. J. M. Verbaarschot, Phys. Rev. D 75, 116003 (2007).
[17] J. Han and M. A. Stephanov, Phys. Rev. D 78, 054507 (2008).

[18] C. R. Allton et al., Phys. Rev. D 66, 074507 (2002).

[19] P. H. Ginsparg, Nucl. Phys. B 170, 388 (1980).

[20] T. Appelquist and R. D. Pisarski, Phys. Rev. D 23, 2305 (1981).

[21] E. Braaten and A. Nieto, Phys. Rev. D 53, 3421 (1996).

[22] K. Kajantie, M. Laine, K. Rummukainen and M. E. Shaposhnikov, Nucl. Phys. B 458, 90 (1996).

[23] A. Hart, M. Laine and O. Philipsen, Nucl. Phys. B 586, $443(2000)$.

[24] A. Vuorinen, Phys. Rev. D 68, 054017 (2003).

[25] A. Ipp, K. Kajantie, A. Rebhan and A. Vuorinen, Phys. Rev. D 74, 045016 (2006).

[26] S. Weinberg, Physica A 96, 327 (1979); Phys. Lett. B 91, 51 (1980).

[27] E. Braaten and R. D. Pisarski, Nucl. Phys. B 337, 569 (1990).

[28] E. Braaten and R. D. Pisarski, Nucl. Phys. B 339, 310 (1990).

[29] J. Frenkel and J. C. Taylor, Nucl. Phys. B 334, 199 (1990).

[30] C. P. Korthals Altes, R. D. Pisarski and A. Sinkovics, Phys. Rev. D 61, 056007 (2000).

[31] S. Ejiri, Phys. Rev. D 77, 014508 (2008); Phys. Rev. D 78, 074507 (2008).

[32] M. Le Bellac Thermal Field Theory (Cambridge University Press, Cambridge, 1996).

[33] P. Pascual and R. Tarrach, Lect. Notes Phys. 194, 1 (1984). 